\title{
Preparation of Borneol Angelica Polysaccharide Liposomes and Study Evaluation of Its Anti-Cerebral Ischemia-Reperfusion Inflammatory Reaction
}

\section{Ke Wang}

Hunan University of Chinese Medicine

Lin Qin He

Hunan University of Chinese Medicine

Xiao Yu Yang

Hunan University of Chinese Medicine

Hai Hong Huang

Hunan University of Chinese Medicine

Wen Long Liu

Hunan University of Chinese Medicine

Chen Xiao

Hunan University of Chinese Medicine

Ze Xuan Du

Hunan University of Chinese Medicine

huang ding

Hunan University of Chinese Medicine https://orcid.org/0000-0001-8628-884X

Wei Zhang ( $\square$ zhangwei1979@hnucm.edu.cn )

Hunan University of Chinese Medicine

\section{Nano Express}

Keywords: borneol, angelica polysaccharide, nanoliposomes, cerebral ischemia-reperfusion, inflammatory reaction

Posted Date: September 30th, 2021

DOI: https://doi.org/10.21203/rs.3.rs-942043/v1

License: (c) (1) This work is licensed under a Creative Commons Attribution 4.0 International License. Read Full License 


\section{Abstract}

Objective To prepare borneol angelica polysaccharide liposomes (BAPLs) with distinct characteristics in Chinese and Western medicine, to explore their effects on anti-cerebral ischemia-reperfusion inflammatory reactions and to explain their mechanism.

Methods Under the guidance of the basic theory of traditional Chinese medicine, a new dosage form of nanoliposomes was selected to prepare BAPL by the thin film dispersion ultrasonic method. The effects of angelica polysaccharide (AP), lecithin, cholesterol and ultrasonication time on encapsulation efficiency were investigated. Triphenyltetrazolium chloride (TTC) staining was used to detect the volume of cerebral infarction. Western blotting was used to detect the expression levels of TLR-4, NF-KBp65, ZO-1, ZO-2, IL-1 $\beta$, IL-6, IL-8 and IL-10.

Results As the mass ratio of lecithin to cholesterol $\left(X_{1}\right)$, the mass of angelica polysaccharide $\left(X_{2}\right)$ and the interaction between $\mathrm{X}_{1}$ and $\mathrm{X}_{2}\left(\mathrm{X}_{1} \mathrm{X}_{2}\right)$ were used as investigation factors, the fitting equation between encapsulation efficiency $\mathrm{Y}$ and $\mathrm{X}$ was $\mathrm{Y}=0.307-0.153 \mathrm{X}_{1}+0.026 \mathrm{X}_{2}+0.006 \mathrm{X}_{1}{ }^{2}-0.000301 \mathrm{X}_{2}{ }^{2}+0.002 \mathrm{X}_{1} \mathrm{X}_{2}(P]$ $0.05)$. The best ultrasonication time was $15 \mathrm{~min}$, the highest encapsulation efficiency was $80.4 \%$, the particle size was $179.1 \mathrm{~nm}$, and the surface zeta potential was $-17.2 \mathrm{mV}$. In BAPL group, the infarct volume of TTC staining was significantly decreased (P凶0.05), and the expression levels of NF-KBp65, TLR-4, IL-8, IL-6, IL-1 $\beta$ in brain tissue were significantly decreased (Pख0.05), while the expression levels of ZO-1, ZO-2, IL-10 were significantly increased (Pख0.05).

Conclusion BAPL has high encapsulation efficiency, small particle size and stable properties and can alleviate cerebral ischemia-reperfusion injury. Its mechanism may be related to inhibiting inflammatory reactions, downregulating inflammatory reaction signaling pathways and protecting the blood-brain barrier (BBB).

\section{Introduction}

Cerebral ischemia-reperfusion injury refers to brain tissue blood supply reduction due to various causes, ischemia-hypoxia and necrosis of brain tissue and aggravation of brain tissue injury when the blood supply is restored ${ }^{[1]}$. When ischemia-reperfusion occurs in brain tissue, there are a large number of inflammatory factors in ischemic foci. Some studies have shown that the activation and infiltration of inflammatory cells and the synthesis and secretion of adhesion molecules present a cascade reaction that enhances and promotes itself. The reaction can also transform brain tissue from ischemic injury to inflammatory injury through specific inflammatory signaling pathways.

The traditional Chinese medicine Angelica sinensis has the effect of nourishing blood and promoting blood circulation and is commonly used in the treatment of cerebral ischemia in the clinic. Angelica polysaccharide (AP) is an effective polysaccharide component extracted from Angelica sinensis, and its monosaccharide components include arabinose, rhamnose, galactose and glucose ${ }^{[2]}$. Studies have shown that AP has an anti-cerebral ischemia-reperfusion inflammatory reaction effect, which is of great 
significance for the treatment of cerebral ischemia-reperfusion injury ${ }^{[3]}$. However, AP has strong water solubility and has difficulty penetrating the blood-brain barrier (BBB); therefore, it is difficult to achieve a good treatment effect. The traditional Chinese medicine borneol can allow the drug to reach the brain and refresh the mind. Studies have shown that borneol can promote the drug permeability of the BBB ${ }^{[4]}$.

Nanoliposomes are the first nanodrug delivery system that has been successfully applied in the clinic. They have the advantages of plastic particle size, good histocompatibility, lower immunogenicity and lower cytotoxicity ${ }^{[5]}$. Liposomes are bilayer lipid molecules that have good compatibility with the BBB and easily penetrate it.

Under the guidance of traditional Chinese medicine theory, with angelica polysaccharide as "the principal drug", borneol and liposomes function as "the adjuvant drugs" for synergistic effects. Combined with the new liposome technology, AP and borneol were encapsulated by liposomes to prepare BAPL. As the carrier, liposomes can improve the liposolubility of AP. Under the synergistic effect of borneol, AP easily penetrates the BBB and can have a better effect in anti-cerebral ischemia-reperfusion inflammatory reactions. This study provides new ideas for the treatment of cerebral ischemic diseases with integrated traditional Chinese and Western medicine and provides new methods for nanomedicine research and the modern application of traditional Chinese medicine. Therefore, the preparation of BAPL and its anticerebral ischemia-reperfusion inflammatory reaction have important research value. The diagram of the synergistic effect between Angelica sinensis and borneol is shown below.

\section{Experimental}

\section{Experimental Reagents}

Lecithin and cholesterol (Shanghai Macklin Biochemical Co., Ltd., No. C11172484, C10927204); Angelica polysaccharide (Shanghai Yuanye Bio-Technology Co., Ltd., No. C28J10Y80124); borneol (L-borneol with $86 \%$ quality fraction, Hubei Junhui Pharmacy Co., Ltd., No. 20190815); triphenyltetrazole chloride (Shanghai Pharmaceutical Group, China, No. 20191018); NF-kBp65, TLR-4, IL-10 (Proteintech Group, Inc., No. 10745-1-AP, 19811-1-AP, 20850-1-AP); ZO-1, ZO-2 (Affinity, Inc., No. AF5145, DF8632); IL-1ß, IL-6, and IL8 primary antibodies (ABclonal Technology Co., Ltd., No. A1112, A0286, A2541); $\beta$-actin primary antibody (Proteintech Group, Inc., America, No. 66009-1-Ig); goat anti-mouse secondary antibody and goat antirabbit secondary antibody (Proteintech Group, Inc., America, No. SA00001-1, SA00001-2); BCA protein quantitation kit; petroleum benzine, concentrated sulfuric acid and phenol were all analytical reagents, absolute ethanol; borneol angelica polysaccharide liposomes, angelica polysaccharide liposomes, and angelica polysaccharide solution were all prepared according to the methods and steps described in 2.1. Benzylpenicillin was prepared for injection with chloral hydrate and pentobarbital.

\section{Experimental Animals}

Male Sprague-Dawley (SD) rats of SPF grade, weighing 180-200 g, were purchased from Hunan Slick Jingda Experimental Animal Co., Ltd., No. SCXK (Xiang) 2019-0004. Experiments in this study were in 
accordance with the Guide on Animal Research of the Chinese Ethics Committee and were provided free diet and water under a $12 \mathrm{~h}$ day-night cycle at an indoor temperature of $26^{\circ} \mathrm{C}$ and a relative humidity of $60 \%$. The animal experiments were approved by the Laboratory Animal Ethics Committee of Hunan University of Chinese Medicine (No. LL2019102503).

\section{Methods}

\subsection{BAPL Preparation and Encapsulation Efficiency Calculation}

\subsubsection{Preparation of BAPL by the Film Dispersion Ultrasonic Method}

Step 1: Lecithin and cholesterol were dissolved in a mixture of $30 \mathrm{~mL}$ of petroleum benzine and $10 \mathrm{~mL}$ of absolute ethanol, and then the mixed liquid was placed in an eggplant-shaped flask to obtain the suspension. Step 2: The suspension obtained in Step 1 was rotated and evaporated to form a light-yellow translucent film at $80 \mathrm{r} / \mathrm{min}$ in a constant temperature water bath at $50{ }^{\circ} \mathrm{C}$. Step 3: Twenty milliliters of phosphate buffer with AP was added to the eggplant-shaped flask to elute and disperse the film. Then, 5 $\mathrm{mg}$ of borneol dissolved in $0.5 \mathrm{~mL}$ of absolute ethanol was added and shaken for $10 \mathrm{~min}$. Step 4: The suspension in Step 3 was placed in a $300 \mathrm{~W}$ probe ultrasonic water bath at $40^{\circ} \mathrm{C}$ for $15 \mathrm{~min}$. Step 5: The suspension in Step 4 was filtered three times with a $0.45-\mu \mathrm{m}$ microporous membrane to prepare the BAPL suspension and was refrigerated at $4{ }^{\circ} \mathrm{C}$ until further use. The flowchart of BAPL preparation is as follows:

\subsubsection{Calculation of the Liposome Encapsulation Efficiency}

(1) Determination of the detection wavelength: $4 \mathrm{~mL}$ of $4 \mathrm{mg} / \mathrm{mL}$ AP solution was drawn accurately, and 5 $\mathrm{mL}$ of concentrated sulfuric acid and $1 \mathrm{~mL}$ of $5 \%$ phenol solution were added and mixed well. The mixed solution was placed in a boiling water bath for $15 \mathrm{~min}$ and cooled with running water. With pure water as the blank control, a Multiscan Spectrum was used to detect the wavelength range of $200 \mathrm{~nm}$ to $800 \mathrm{~nm}$, and the absorption value was the maximum at $490 \mathrm{~nm}$; therefore, the detection wavelength was determined to be $490 \mathrm{~nm}^{[6]}$.

(2) Preparation of the standard curve: The standard curve was prepared with glucose standard substance. Ten milligrams of glucose standard was weighed precisely and dissolved in pure water. Then, a volume of $100 \mathrm{~mL}$ was made constant to obtain a $1 \mathrm{mg} / \mathrm{mL}$ glucose solution. Then, $10 \mathrm{~mL}$ of glucose solution was drawn from it and diluted to $100 \mathrm{~mL}$ with water to obtain the $0.1 \mathrm{mg} / \mathrm{mL}$ glucose standard solution. Glucose standard solutions of $0,0.2,0.4,0.6,0.8,1.0$ and $1.2 \mathrm{~mL}$ were drawn into centrifuge tubes, and pure water was added until $2 \mathrm{~mL}$ was reached. Then, $5 \mathrm{~mL}$ of concentrated sulfuric acid and $1 \mathrm{~mL}$ of $5 \%$ phenol solution were added to each tube and mixed well. All mixed tubes were placed in a boiling water bath for $15 \mathrm{~min}$ and cooled with running water. After cooling, the absorbance was detected at $490 \mathrm{~nm}$ with a Multiscan Spectrum ${ }^{[7]}$. The glucose concentration $(X)$ was used to regress the absorbance $(Y)$ to obtain the regression equation: $Y=0.041 X+0.12, R^{2}=0.998$. The results showed that glucose had a good linear relationship in the range of $2.5 \bigotimes 15 \mu \mathrm{g} / \mathrm{mL}$. 
1. Determination of encapsulation efficiency: Four milliliters of prepared BAPL suspension was absorbed and centrifuged in a chilled ultracentrifuge $\left(4^{\circ} \mathrm{C}, 10,000 \mathrm{r} / \mathrm{min}, 30 \mathrm{~min}\right)$. The supernatant was extracted in a 50-mL volumetric flask, diluted with pure water to $50 \mathrm{~mL}$ and then mixed well. Four milliliters was accurately drawn into a $10-\mathrm{ml}$ centrifuge tube. Five milliliters of concentrated sulfuric acid and $1 \mathrm{~mL}$ of $5 \%$ phenol solution were added and mixed. The solution was then heated in boiling water for $15 \mathrm{~min}$ and cooled with running water. After cooling, $100 \mu \mathrm{L}$ was dropped into 96 -well plates, and $100 \mu \mathrm{L}$ of pure water was added to dilute each plate. The absorbance was detected with a Multiscan Spectrum, and the results were matched to the standard curve to calculate the concentration of free AP and obtain the mass of free AP from the concentration and ultimately to calculate the encapsulation efficiency. The formula for the encapsulation efficiency was encapsulation efficiency $=\left(1-A_{\text {free }} / A_{\text {total }}\right) \times 100 \%$, where $A_{\text {free }}$ was the mass of free AP and $A_{\text {total }}$ was the mass of added AP when preparing BAPL.

2. The flow chart of BAPL encapsulation efficiency determination is shown below.

\subsubsection{Determination of Borneol}

According to the existing liposome preparation process, $3 \otimes 10 \mathrm{mg}$ of borneol was added as an adjuvant drug. Combined with the previous experiments in this study, it was found that the encapsulation efficiency of borneol was the highest when the mass of borneol was $5 \mathrm{mg}$; therefore, the mass of borneol used in this study was $5 \mathrm{mg}$.

\subsubsection{Measurement of BAPL Particle Size and Surface Potential}

(1) Measurement of liposome particle size: A 1-mL sample of BAPL suspension was slowly added into the sample tank with a 1-mL syringe and inserted into the groove. The DTS software was opened, and the number of measurements was set to 3 . Then, the test was initiated, and the results were automatically saved. (2) Zeta potential test on the liposome surface: BAPL suspension was added to the side of the sample tank with a syringe until the sample was filled in a U-tube and inserted into the groove. The DTS software was opened, and the number of measurements was set to 3 . Then, the test was initiated, and the results were automatically saved.

\subsubsection{Electron Microscope Observation of Liposomes}

A total of $10 \mu \mathrm{L}$ of sample suspension was dropped on a copper mesh carrier with film. The sample was negatively stained with $2 \%$ phosphotungstic acid and dried in a static state. The microscopic morphology of BAPL was observed by transmission electron microscopy.

\subsubsection{Statistical Analysis}

The experimental results are expressed as ${ }^{\prime} \mathrm{c} \pm \mathrm{s}$ and were analyzed with SPSS 20.0 software. After quadratic regression analysis, the fitting equation of the relationship between encapsulation efficiency $Y$ and influencing factor $X$ was obtained. $P<0.05$ indicated that the difference was statistically significant. 


\subsection{Study of the Anti-Cerebral Ischemia-Reperfusion Inflammatory Reaction of BAPL}

In this study, a cerebral ischemia-reperfusion rat model was designed as the research object. TTC staining and Western blot analysis were used to explore the effect and mechanism of BAPL in the anti-cerebral ischemia-reperfusion inflammatory reaction.

\subsubsection{Groupings}

The SD rats were fed for one week, weighed and then divided into 5 groups with 5 rats in each group: the BAPL group (cerebral ischemia-reperfusion model with BAPL suspension administration); the APL group (cerebral ischemia reperfusion model with APL suspension administration); the AP group (cerebral ischemia reperfusion model with AP administration); the Model group (cerebral ischemia reperfusion model with saline administration); and the Sham group, which was given saline.

\subsubsection{Drug Administration and Model Preparation}

The SD rats were fed adaptively for 7 days followed by tail vein injection once a day for 3 days (BAPL suspension, APL suspension and AP solution were injected at a single dose of $30 \mathrm{mg} / \mathrm{kg} \mathrm{AP)}$. The rats were modeled 2 hours after the last administration, and the rats were fasted 12 hours before the operation. The cerebral ischemia-reperfusion rat model was established by the suture method. The Sham group underwent the same operation without inserting a suture through the common carotid artery. After cerebral ischemia in rats was established, penicillin was used to avoid infection, and then the skin was sutured. After 2 hours of ischemia, the rats were anesthetized again, and the sutures were pulled out to restore the blood supply of the left common carotid artery and left internal carotid artery; thus, the rat model of cerebral ischemia was established for 2 hours, followed by reperfusion for 24 hours. During reperfusion, the drug administration schedule was the same as before. During the operation, the rats were continuously heated with a heating blanket and incandescent lamp to maintain their rectal temperature at $37.5 \pm 0.3^{\circ} \mathrm{C}$. The dry bedding materials were paved at 3 to $5 \mathrm{~cm}$ height. After the operation, the rats were placed in a thermostat until they awakened. Then, the rats were moved to a feeding box paved with 3 to $5 \mathrm{~cm}$ dry bedding materials ${ }^{[8]}$.

\subsubsection{Scores of Neurological Function}

The neurological deficits of rats were scored by Zea-Longa's score. The scores ranged from 0 to 4 points. The higher the score, the more severe the neurological deficits were ${ }^{[9]}$. The standards of Zea-Longa's scores are shown in Table 1.

\section{Table 1 Five-grade Standard Scores by Zea-Longa}




\begin{tabular}{|l|}
\hline Behavioral and Psychological Symptoms Points \\
Normal movement; when lifting the tail, the two fore claws can \\
straighten to the ground without neurological deficit. \\
When lifting the tail, the right fore claw cannot straighten \\
completely, and the rats circle around to the right when crawling. \\
When lifting the tail, the right fore claw is incurvate, and \\
the rats circle around to the right when crawling. \\
\hline Stand unsteadily and fall to hemiplegia side when crawling. \\
\hline Conscious disorder and unable to walk spontaneously.
\end{tabular}

\subsubsection{Observation of Cerebral Infarction Volume by TTC Staining}

After ischemia for 2 hours and reperfusion for 24 hours, pentobarbital was injected intraperitoneally into the rats, and brain tissue was collected after death. TTC staining was used to observe cerebral infarction: 3 rats were randomly selected in each group. Their brain tissue was removed by decapitation and placed in a refrigerator at $-20^{\circ} \mathrm{C}$ for several minutes, and then the olfactory bulb, cerebellum and low brainstem were removed. Five coronary slices were cut from the frontal pole to the occipital pole, immersed in $2 \%$ TTC phosphate buffer, stained in the dark at $37^{\circ} \mathrm{C}$ for $30 \mathrm{~min}$, fixed with $4 \%$ formaldehyde solution for 30 min and photographed. The white part was the infarct area, while the red part was the normal brain tissue. Image-Pro Plus 6.0 software was used to analyze the cerebral infarction area of rats in each group. The calculation formula was $\mathrm{V}=\mathrm{A}(\mathrm{B} 1+\mathrm{B} 2 \ldots \ldots+\mathrm{Bn})$ for the calculation of cerebral infarction volume in rats, and its percentage to the whole rat brain was further calculated, where $A$ was the slice thickness and $B$ was the infarct area.

\subsubsection{Western-Blot Analysis of Protein Expression}

The rat brain tissues from the infarction area were removed and repeatedly ground at low temperature to obtain brain tissue homogenates. After adding the appropriate RIPA lysate, the supernatant was collected by centrifugation at $4{ }^{\circ} \mathrm{C}$. The total protein concentration was measured by the BCA method. The sample was loaded with $20 \mu \mathrm{g}$ of total protein, subjected to constant voltage electrophoresis and then transferred to a PVDF membrane. After sealing and rinsing, the IL-1 $\beta$ (1:1000), IL-6 (1:1000), IL-8 (1:1000), IL-10 (1:1000), NF-KBp65 (1:2000), TLR-4 (1:750), ZO-1 (1:5000), ZO-2 (1:5000), and $\beta$-a primary antibodies (1:5000) were added and incubated overnight and were then washed 3 times with TBST for 15 min each time. Then, goat anti-rabbit secondary antibody (1:6000) or goat anti-mouse secondary antibody (1:5000) was added, incubated at $37^{\circ} \mathrm{C}$ for 1 hour and washed 3 times with TBST for 10 min each time. ECL chemiluminescence agent was added to the gel image analysis system, and the exposed films were scanned. Quantity One software was used for professional gray analysis, and the relative values of the target proteins were obtained from the ratio of the gray levels between the IL-1 $\beta$, IL-6, IL-8, IL-10, NF-KBp65, TLR-4, ZO-1, ZO-2 and $\beta$-actin protein bands. 


\section{Results}

\section{Determination of the Optimal Ultrasonic Time}

The BAPL was prepared following the method described in 2.1. Its encapsulation efficiency was measured, and the liposome encapsulation ratio was used as the response to investigate the effects of ultrasonication time on the encapsulation efficiency of BAPL at $5,10,15,20$, and $25 \mathrm{~min}$. The results showed that the encapsulation efficiency of BAPL changed with ultrasonication time. To some extent, the encapsulation efficiency of BAPL was reduced at ultrasonication times that were too long or too short. Figure 5 shows that when the ultrasonication time was $15 \mathrm{~min}$, the encapsulation efficiency of BAPL reached its maximum, and when the ultrasonication time was extended successively, the encapsulation efficiency of BAPL decreased slightly. Considering the encapsulation efficiency index, the ultrasound time of BAPL preparation in this study was set as $15 \mathrm{~min}$.

\section{Uniform Design to Screen the Optimal Combination}

Based on preliminary research, the masses of lecithin, cholesterol and AP were the three major factors that had significant influences on the BAPL encapsulation efficiency. The three factors were chosen as the uniform design factors, and the encapsulation efficiency was used as the response. Therefore, the uniform design of the three factors and seven levels was arranged, and each group was repeated four times. The level of each factor is shown in Table 2.

\section{Table 2 Factor Levels}

\section{Analysis of the Uniform Design Results}

\begin{tabular}{|c|c|c|c|}
\hline Testing Number & Lecithin $\rrbracket \mathrm{mg} \rrbracket$ & Cholesterol $₫ \mathrm{mg} \rrbracket$ & $\mathrm{AP} \otimes \mathrm{mg} \rrbracket$ \\
\hline 1 & 120 & 60 & 45 \\
\hline 2 & 210 & 30 & 40 \\
\hline 3 & 60 & 80 & 35 \\
\hline 4 & 150 & 50 & 30 \\
\hline 5 & 240 & 20 & 25 \\
\hline 6 & 90 & 70 & 20 \\
\hline 7 & 180 & 40 & 15 \\
\hline
\end{tabular}

The liposomes were prepared by the method described in 2.1, and the encapsulation efficiency of each group was measured. The analysis of uniform design results were as follows: $X_{1}$ (the mass and membrane material ratio of lecithin and cholesterol), $X_{2}$ (the mass of $A P$ ), $X_{1} X_{2}$ (the interaction of membrane material ratio and AP mass) were designed as the influencing factors, the encapsulation efficiency was used as the response, and through quadratic regression analysis, the fitting equation of the encapsulation rate $Y$ and 
the influencing factors $X$ was determined to be $Y=0.307-0.153 X_{1}+0.026 X_{2}+0.006 X_{1}{ }^{2}$ -

$0.000301 X_{2}{ }^{2}+0.002 X_{1} X$. From the result of the fitting equation model, it can be seen that the fitting model equation is highly significant $\left(P_{\text {model }}<0.05\right)$. In addition, in this model, the $\mathrm{P}$ values of the three influencing factors were all less than 0.05 , indicating that they had a significant impact on the encapsulation efficiency of BAPL. The multiple correlation coefficient $\mathrm{R}=0.994$, the determination coefficient $R^{2}=0.988$ and the correction determination coefficient $R_{\text {pred }}{ }^{2}=0.985$ were consistent with $R 2$. In conclusion, this equation has a good fitting degree. The uniform design results are shown in Table 3.

Table 3 Uniform Design Results of Each Group ( $c \pm s, n=4$ )

\begin{tabular}{|ll|}
\hline Testing Number & Encapsulation Rate \\
\hline 1 & $75.375 \% \pm 1.9906 \%$ \\
\hline 2 & $63.550 \% \pm 2.9738 \%$ \\
\hline 3 & $77.450 \% \pm 0.3109 \%$ \\
\hline 4 & $58.575 \% \pm 3.5743 \%$ \\
\hline 5 & $42.225 \% \pm 2.6887 \%$ \\
\hline 7 & $56.800 \% \pm 2.2136 \%$ \\
\hline
\end{tabular}

\section{Experimental Verification on the Fitting Equation}

Based on the method described in 2.1, the BAPL was prepared as Case 1, Case 2 and Case 3 to verify the predicted accuracy of the fitting equation. The results are shown in Table 4. Since this study mainly focused on the effects of lecithin mass, cholesterol mass and AP mass on encapsulation efficiency, the effects of other factors were not investigated. Therefore, there was an error (approximately $5 \%$ ) between the predicted encapsulation efficiency and the measured encapsulation efficiency. The fitting degree detection of the multiple correlation coefficient was $R^{2}=0.988$, the correction determination coefficient was $R_{\text {pred }}{ }^{2}=0.985$, and the multiple correlation coefficient was $R=0.994$; therefore, the equation fitted well. According to the existing experimental data, the combination of Case 1 had the highest encapsulation efficiency. Therefore, the subsequent preparation of BAPL in this study was based on the ratio of lecithin to AP mass (lipid-drug ratio), the membrane material and the borneol mass in Case 1.

Table 4 Verification of the predicted accuracy of the Fitting Equation 


\begin{tabular}{|lllllll|}
\hline $\begin{array}{l}\text { Case } \\
\text { Number }\end{array}$ & Lecithin & Cholesterol & AP & Borneol & $\begin{array}{l}\text { Encapsulation } \\
\text { Efficiency(predicted) }\end{array}$ & $\begin{array}{l}\text { Encapsulation } \\
\text { Efficiency(measured) }\end{array}$ \\
\hline Case 1 & $60 \mathrm{mg}$ & $60 \mathrm{mg}$ & $45 \mathrm{mg}$ & $5 \mathrm{mg}$ & $81 \%$ & $80.4 \%$ \\
\hline Case 2 & $80 \mathrm{mg}$ & $60 \mathrm{mg}$ & $45 \mathrm{mg}$ & $5 \mathrm{mg}$ & $76.5 \%$ & $71.0 \%$ \\
\hline Case 3 & $120 \mathrm{mg}$ & $60 \mathrm{mg}$ & $45 \mathrm{mg}$ & $5 \mathrm{mg}$ & $73.2 \%$ & $70.6 \%$ \\
\hline
\end{tabular}

\section{The Particle Size Distribution and Surface Potential of BAPL}

The particle size distribution of BAPL showed that the particle size of BAPL was equally distributed, with an average particle size of $179.1 \mathrm{~nm}$ (average of 3 measurements), and the surface zeta potential was $-17.2 \mathrm{mV}$ (average of 3 measurements). The particle size distribution of BAPL is shown in Figure 6.

\section{Electron Microscope Results of BAPL}

The BAPL prepared according to Case 1 (the highest encapsulation efficiency combination mentioned in 3.4) showed bilayer lipid molecules of liposomes under an electron microscope, which were spherical or quasi-spherical, and the size distribution of liposomes was relatively uniform. The electron microscopic results are shown in Figure. 7.

\section{Results of Zea-Longa's Score}

After cerebral ischemia for 2 hours and reperfusion for 24 hours, the neurological function of rats was scored. Rats with scores of 1-3 were chosen for the follow-up experiments, while rats with scores of 0 points and 4 points were discarded. The results are shown in Figure 8.

\section{Results of TTC Staining}

Red indicates normal brain tissue, and gray indicates the infarction area. The TTC staining results showed that cerebral infarction increased significantly after cerebral ischemia and reperfusion. Compared with the model group, the cerebral infarction area in the BAPL group decreased $(P<0.01)$ and was smaller than those in the APL and AP groups.

\section{Results of Western-Blot Analysis}

The expression levels of four inflammatory factors, IL-10, IL-8, IL- 6 and IL-1 $\beta$, were analyzed. The results showed that the expression of IL-10 decreased while the expression of IL-8, IL- 6 and IL-1 $\beta$ increased after cerebral ischemia-reperfusion injury. Compared with that in the model group, the expression of IL-10 in the BAPL group was increased $(P<0.01)$ and was higher than those in the APL and AP groups $(P<0.01)$. The expression levels of IL-8, IL- 6 and IL-1 $\beta$ in the BAPL group were decreased $(P<0.01)$ and were lower than those in the APL and AP groups $(P<0.05$ or $P<0.01)$.

The expression levels of four proteins, NF-KBp65, TLR-4, ZO-1 and ZO-2, were detected. In the model group, the results showed that the protein expression of NF-KBp65 was decreased $(P<0.01)$ and was lower than 
those in the APL and AP groups $(\mathrm{P}<0.05$ or $\mathrm{P}<0.01)$; the protein expression of TLR-4 was decreased $(P<0.01)$ and was lower than those of the APL and AP groups $(P<0.01)$, while the protein expression levels of ZO-1 and ZO-2 were increased $(P<0.01)$ and were higher than those of the APL and AP groups $(P<0.01)$.

\section{Discussion}

Liposomes are spherical liposomes with bilayer lipid molecules formed by hydration and ultrasound of lecithin and cholesterol in organic solvents. Liposomes have a cell-like structure, which can not only improve the effective drug concentration in target lesions by changing the distribution of encapsulated drugs in vivo but also limit the effects of encapsulated drugs on normal tissues and organs to a certain extent to improve drug efficacy and reduce drug toxicity. At present, there are many methods for liposome preparation, which have advantages and disadvantages. For example, the formation of liposomes is incomplete by the film dispersion method, and the melting method results in lower drug encapsulation by reverse evaporation and is difficult to perform ${ }^{[10]}$. In this study, the film dispersion ultrasonic method was used to prepare BAPL with a small amount of borneol added to enhance the targeting of BAPL and promote its penetrability across the BBB. The BAPL prepared by this method had a high encapsulation efficiency, small and uniform distributed particle size, and stable surface zeta potential. In this study, the encapsulation efficiency of BAPL was calculated by the ultracentrifugation method and the phenol-sulfuric acid method. Their operation was simple, and the separating effect between free AP and BAP was better. By measuring free AP, the encapsulation efficiency of BAPL can be measured more accurately. It is generally believed that increasing the lipid-drug ratio can improve the encapsulation efficiency of liposomes. However, this study found that with an increase in the lipid-drug ratio, the encapsulation efficiency did not increase. This may be related to the liposomes that encapsulated AP and borneol reaching a saturation state. The model equation was obtained by analyzing the results of uniform design. Through this model, the encapsulation efficiency of BAPL was predicted accurately, and the preparation process parameters of BAPL were optimized. In this study, the optimal preparation parameters were as follows: the membrane material ratio was 1:1, the lipid-drug ratio was 4:3, the ultrasonication time was 15 min and the highest encapsulation efficiency of BAPL was $80.4 \%$. The liposomes are light yellow suspensions with spherical or quasi-spherical morphology, bilayer lipid structure, $179.1 \mathrm{~nm}$ average particle size and $-17.2 \mathrm{mV}$ surface zeta potential, indicating that liposomes, as a new type of nanomaterial, are feasible to encapsulate AP and borneol. Cerebral ischemia-reperfusion injury is an extremely complex process involving various pathophysiological cascades ${ }^{[11]}$. In recent years, an increasing number of scholars have focused on the effects of inflammatory reactions in cerebral ischemia-reperfusion injury, and inflammatory reactions play an important role in the process of brain tissue injury caused by cerebral ischemia-reperfusion. Studies have shown that a large number of inflammatory factors are released after cerebral ischemia-reperfusion, promoting the release of a large number of inflammatory cytokines from platelets, cerebrovascular endothelial cells and intracerebral glial cells ${ }^{[12]}$. Therefore, reducing inflammatory factor release and alleviating the inflammatory reaction of cerebral ischemia-reperfusion injury are important for the treatment of cerebral ischemia-reperfusion disease. 
Traditional Chinese medicine in the treatment of cerebral ischemia disease has attracted increasing attention from scholars. Under the guidance of traditional Chinese medicine theory, how to use traditional Chinese medicine or its components to alleviate cerebral ischemia-reperfusion inflammatory reactions has become the focus of research. Therefore, in this study, a new type of BAPL was prepared by nanotechnology under the guidance of traditional Chinese medicine theory, and its effect on cerebral ischemia-reperfusion injury was studied. The results showed that compared with the Sham group, the neurological function scores of rats in the Model group were significantly increased, and the volume of the cerebral infarction was increased, indicating that the modeling was successful. Compared with that in the model group, the volume of cerebral infarction in the BAPL group was significantly decreased and was lower than those in the APL and AP groups. This result indicated that BAPL could better restore the blood supply of brain tissue and effectively reduce the volume of cerebral infarction, which had a stronger effect than APL and AP.

IL-1 $\beta$ is an important proinflammatory mediator that is mainly produced by mononuclear macrophages. High expression of IL-1 $\beta$ promotes the occurrence of inflammatory reactions ${ }^{[13]}$. During cerebral ischemia, the expression of IL-6 increases significantly, acting on vascular endothelial cells and eventually leading to a large amount of leukocyte aggregation and adhesion, thereby mediating the inflammatory cascade and further aggravating cerebral ischemia damage ${ }^{[14]}$. IL-8 is a chemotactic cytokine of the inflammatory reaction that can induce morphological changes and chemotaxis, release granule inclusions and upregulate adhesion proteins, thereby promoting inflammatory reactions ${ }^{[15]}$. In this study, the results showed that the expression levels of IL-1 $1 \beta$, IL- 6 , and IL-8 in the BAPL group were decreased compared with those in the model group and lower than those in the APL and AP groups, which indicated that during cerebral ischemia-reperfusion injury, BAPL reduced the cerebral ischemia-reperfusion inflammatory reaction by downregulating the expression levels of IL- $1 \beta$, IL- 6 and IL-8, and the effect was stronger than those of APL and AP. IL-10, which is mainly secreted by macrophages and monocytes in tissues, is an important anti-inflammatory factor in vivo that has a neuroprotective effect and inhibits the expression and activation of cytokine receptors ${ }^{[16]}$.

In this study, the results showed that the expression of IL-10 in the BAPL group was increased compared with that in the model group and was higher than those in the APL and AP groups, indicating that in cerebral ischemia-reperfusion injury, BAPL upregulated the expression of IL-10 to resist inflammatory reactions and reduce nerve injury, and the effect was stronger than those of APL and AP. This may be one of the reasons why BAPL results in an anti-cerebral ischemia-reperfusion inflammatory reaction.

In cerebral ischemia-reperfusion injury, the NF-KBp65 and TLR-4 signaling pathways mediate the production and release of a variety of inflammatory mediators and metalloproteinases ${ }^{[17-18]}$. NF-KBp65 is the center of the inflammatory reaction and the intracellular signal transduction pathway ${ }^{[19]}$, and TLR-4 can also recognize the production of mediating inflammatory reactions by lipid peroxide molecules, oxygen free radicals, hydroxyl free radicals and other oxidative stresses ${ }^{[20]}$. In this study, the protein expression levels of NF-KBp65 and TLR-4 in the BAPL group decreased, indicating that BAPL can improve the inflammatory reaction of cerebral ischemia-reperfusion, which may be related to the inhibition of the 
NF-KBp65 and TLR-4 signaling pathways. The ZO-1 protein, as an important structural and functional protein of BBB tight junctions, connects transmembrane proteins and plays an important role in maintaining the polarity of epithelial cells, forming the cytoskeleton and cell tight junctions ${ }^{[21]}$. ZO-1 and ZO-2 are fixed in the cytoplasmic side of the tight junctions between cells in a compound manner and play important roles in maintaining the function of the $\mathrm{BBB}^{[22]}$. After cerebral ischemia-reperfusion injury, the protein expression levels of ZO-1 and ZO-2 are closely related to the degree of BBB injury, and the destruction of its function and structure will cause the dissociation of tight junctions. In this study, the protein expression levels of ZO- 1 and ZO-2 in the BAPL group were increased, which played an important role in maintaining the function of the BBB to reduce cerebral ischemia-reperfusion injury.

\section{Conclusions}

In conclusion, BAPL can alleviate cerebral ischemia-reperfusion injury, which is related to its antiinflammatory reaction. As a new nanomaterial, BAPL can provide novel ideas for the treatment of cerebral ischemia-reperfusion injury.

\section{Declarations}

Availability of data and materials $₫$ All data generated or analysed during this study are included in this published article.

Competing interests $\unrhd$ The authors declare that they have no competing interests.

Funding $₫$ National Natural Science Foundation of China (81774032 82174218); Natural Science Outstanding Youth Fund of Hunan Provincial (2020JJ2024); Natural Science Youth Fund of Hunan Provincial (2018JJ3391, 2020JJ5412); Key scientific research project of Hunan Provincial Department of Education (19A374).

Authors' contributions $\rrbracket$

ZW contributed to the contents of the study;

WK, HLQ, YXY, HHH, DZX performed the experiment;

ZW, WK, LWL contributed significantly to analysis and manuscript preparation;

WK, HLQ, YXY performed the data analyses;

WK, XC, DH wrote the manuscript;

ZW, WK helped perform the analysis with constructive discussions.

All authors read and approved the final manuscript. 
Acknowledgements $₫$ Acknowledged Deng Changqing and Zhou Xudong of Hunan University of Chinese medicine and Wang Yang of Central South University who contributed towards the article.

\section{References}

1. Yang QW, Huang QY, Hu ZP, et al. Potential Neuroprotective Treatment of Stroke: Targeting Excitotoxicity, Oxidative Stress, and Inflammation[J]. Front Neurosci, 2019, 13: 1036.

2. Zhou RM, Ci MX, Wang Yu, et al. Isolation, structure identification and anti-inflammatory activity of a polysaccharide from Phragmites rhizoma[J]. Int J Biol Macromol, 2020, 161: 810-817.

3. Zhang S, He B, Ge J, et al. Extraction, chemical analysis of Angelica sinensis polysaccharides and antioxidant activity of the polysaccharides in ischemia-reperfusion rats[ $\mathrm{J}]$. Int $\mathrm{J}$ Biol Macromol, 2010, 47(4): $546-50$.

4. Chen ZX, Xu QQ, Shan Chun-Shuo, et al. Borneol for Regulating the Permeability of the Blood-Brain Barrier in Experimental Ischemic Stroke: Preclinical Evidence and Possible Mechanism[J]. Oxid Med Cell Longev, 2019, 2019: 2936737.

5. Zhang W, Ma W, Zhang J, et al. The immunoregulatory activities of astragalus polysaccharide liposome on macrophages and dendritic cells[J]. Int J Biol Macromol, 2017, 105(Pt1): 852-861.

6. Zhang W, Zhang M, Cheng A, et al. Immunomodulatory and antioxidant effects of Astragalus polysaccharide liposome in large yellow croaker (Larimichthys crocea)[J]. Fish Shellfish Immunol, 2020, 100: 126-136.

7. Fan Y, Ma L, Zhang W, [J]. Liposome can improve the adjuvanticity of astragalus polysaccharide on the immune response against ovalbumin[J]. Int J Biol Macromol, 2013, 60: 206-12.

8. Tang $Y$, Wang J, Lin $X$, et al. Neural stem cell protects aged rat brain from ischemia-reperfusion injury through neurogenesis and angiogenesis[J]. J Cereb Blood Flow Metab, 2014, 34(7): 1138-47.

9. Longa EZ, Weinstein PR, Carlson S, et al. Reversible middle cerebral artery occlusion without craniectomy in rats[J]. Stroke, 1989, 20(1): 84-91.

10. Zhang XP, Li YD, Luo LL, et al. Astragalus Saponins and Liposome Constitute an Efficacious Adjuvant Formulation for Cancer Vaccines[J]. Cancer Biother Radiopharm, 2018, 33(1): 25-31.

11. Lin L,Wang X, Yu Z. Ischemia-reperfusion Injury in the Brain: Mechanisms and Potential Therapeutic Strategies[J]. Biochem Pharmacol (Los Angel), 2016, 5(4): 213.

12. Chen YK, Zhang L, Ni JS, et al. LLDT-8protects against cerebral ischemia/reperfusion injury by suppressing post-stroke inflammation[J]. Journal of Pharmacological Sciences, 2016, 131(2): 131137.

13. Rizzo FR, Musella A, De Vito F, et al. Tumor Necrosis Factor and Interleukin-1 $\beta$ Modulate Synaptic Plasticity during Neuroinflammation[J]. Neural Plast, 2018, 2018: 8430123.

14. Adibhatla RM, Dempsy R, Hatcher JF. Integration of cytokine biology and lipid metabolism in stroke[J]. Front Biosci, 2008, 13: 1250-70. 
15. Qin $L$, Tao $Y$, Ting $L$, et al. Ginkgetin exerts anti-inflammatory effects on cerebral ischemia/reperfusioninduced injury in a rat model via the TLR4/NF-KB signaling pathway[J]. Biosci Biotechnol Biochem, 2019, 83(4): 675-683.

16. Wang YS, Li YX, Zhao P, et al. Anti-inflammation Effects of Oxysophoridine on Cerebral IschemiaReperfusion Injury in Mice[J]. Inflammation, 2015, 38(6): 2259-68.

17. Yu L, Chen $C$, Wang LF, et al. Neuroprotective effect of kaempferol glycosides against brain injury and neuroinflammation by inhibiting the activation of NF-KB and STAT3 in transient focal stroke[J]. PLoS One, 2013, 8(2): e55839.

18. Chen J, Yang $C$, Xu X, et al. The effect of focal cerebral ischemia-reperfusion injury on TLR4 and NFKB signaling pathway[J]. Exp Ther Med, 2018, 15(1): 897-903.

19. Liang W, Lin C, Yuan L, et al. Preactivation of Notch1 in remote ischemic preconditioning reduces cerebral ischemia-reperfusion injury through crosstalk with the NF-KB pathway[J]. J Neuroinflammation, 2019, 16(1): 181.

20. Wang LJ, Liu HY, Zhang LG, et al. Neuroprotection of Dexmedetomidine against Cerebral IschemiaReperfusion Injury in Rats: Involved in Inhibition of NF-KB and Inflammation Response[J]. Biomol Ther (Seoul), 2017, 25(4): 383-389.

21. Nico B, Ribatti D. Morphofunctional aspects of the blood-brain barrier[J]. Curr Drug Metab. 2012, 13(1): 50-60.

22. Bauer $\mathrm{H}$, Zweimueller-Mayer J, Steinbacher $\mathrm{P}$, et al. The dual role of zonula occludens (ZO) proteins[J]. J Biomed Biotechnol, 2010, 2010: 402593.

\section{Figures}

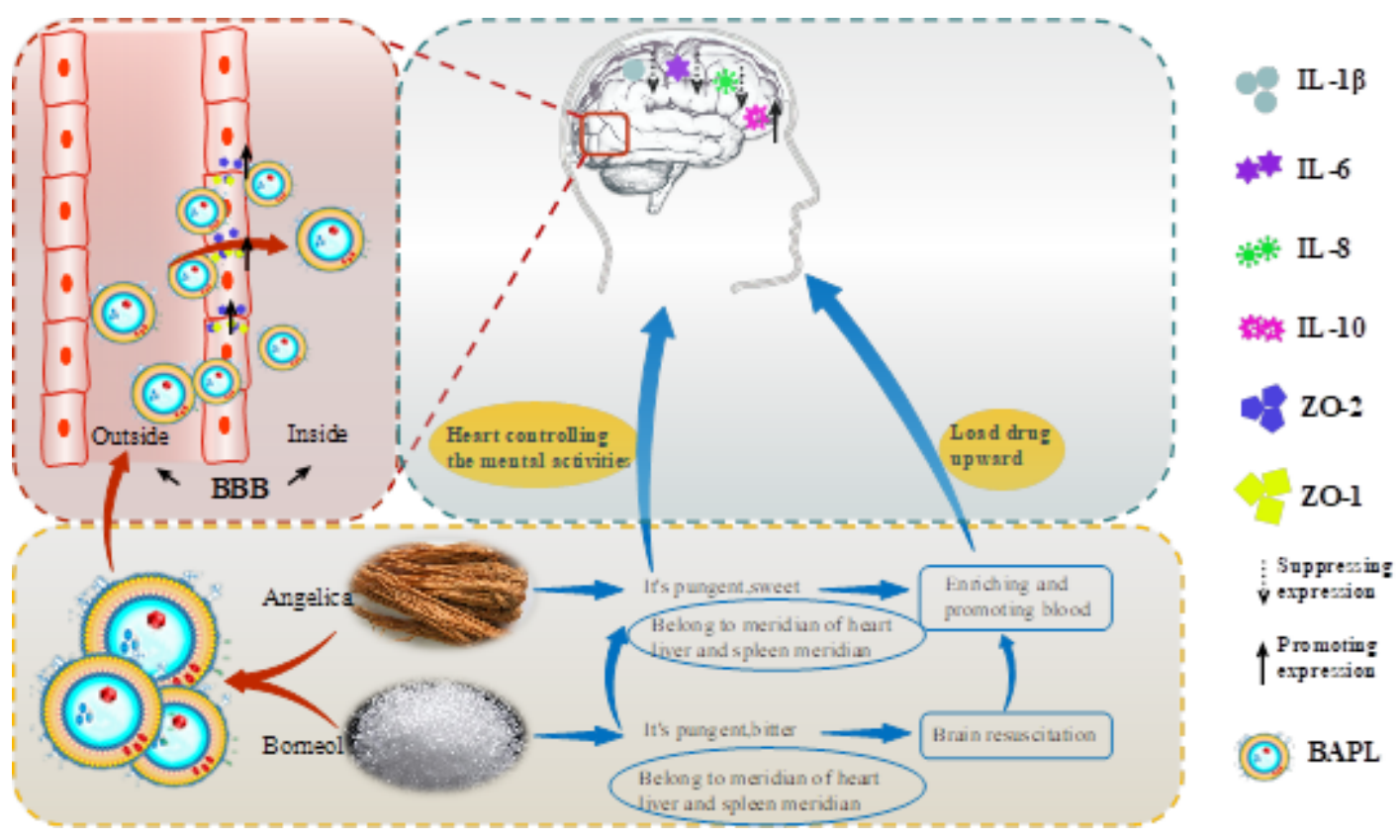

Figure 1 
Diagram of the Synergistic Effect between Angelica sinensis and Borneol

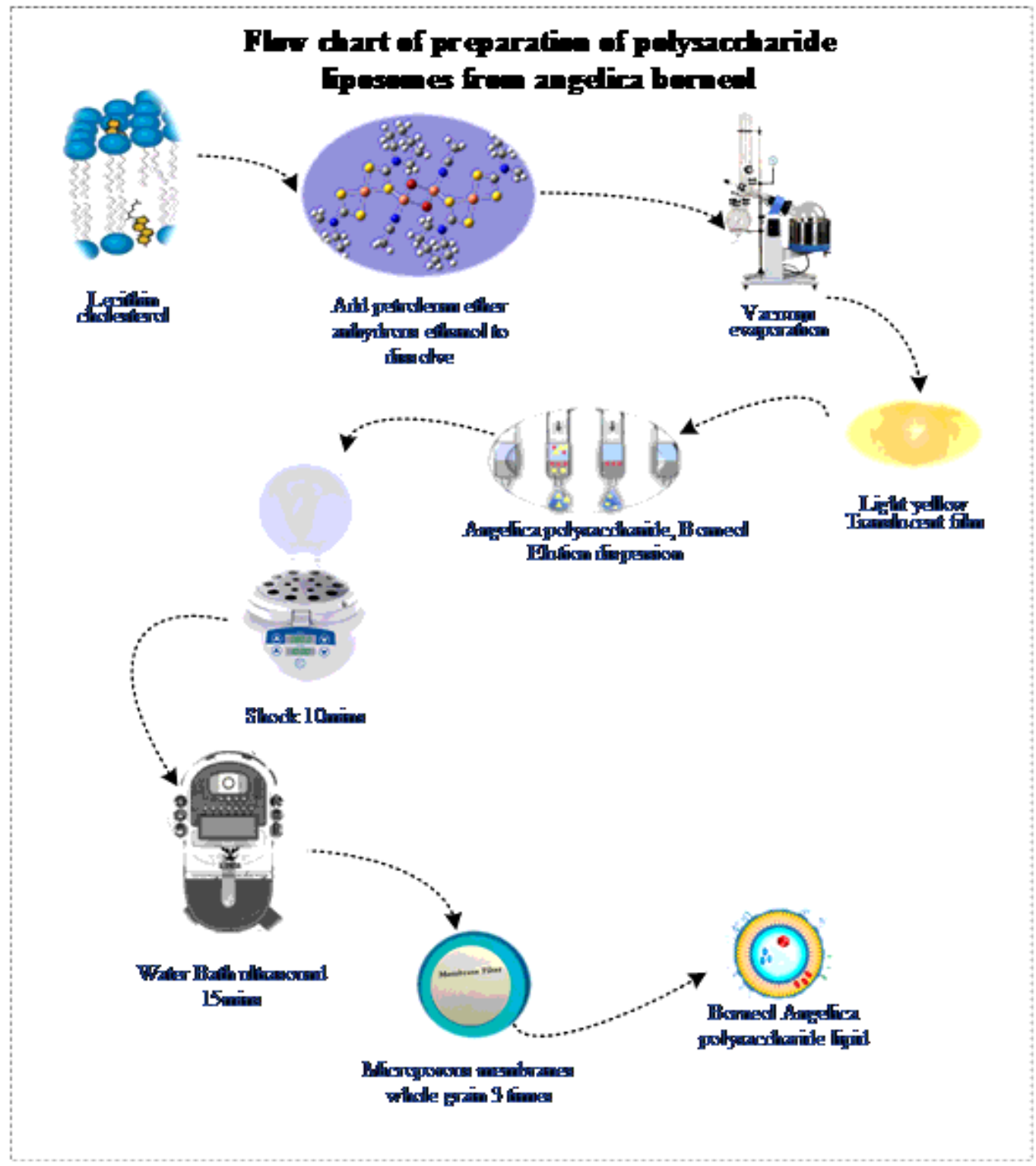

Figure 2

Flowchart of BAPL Preparation 


\section{standard curve}

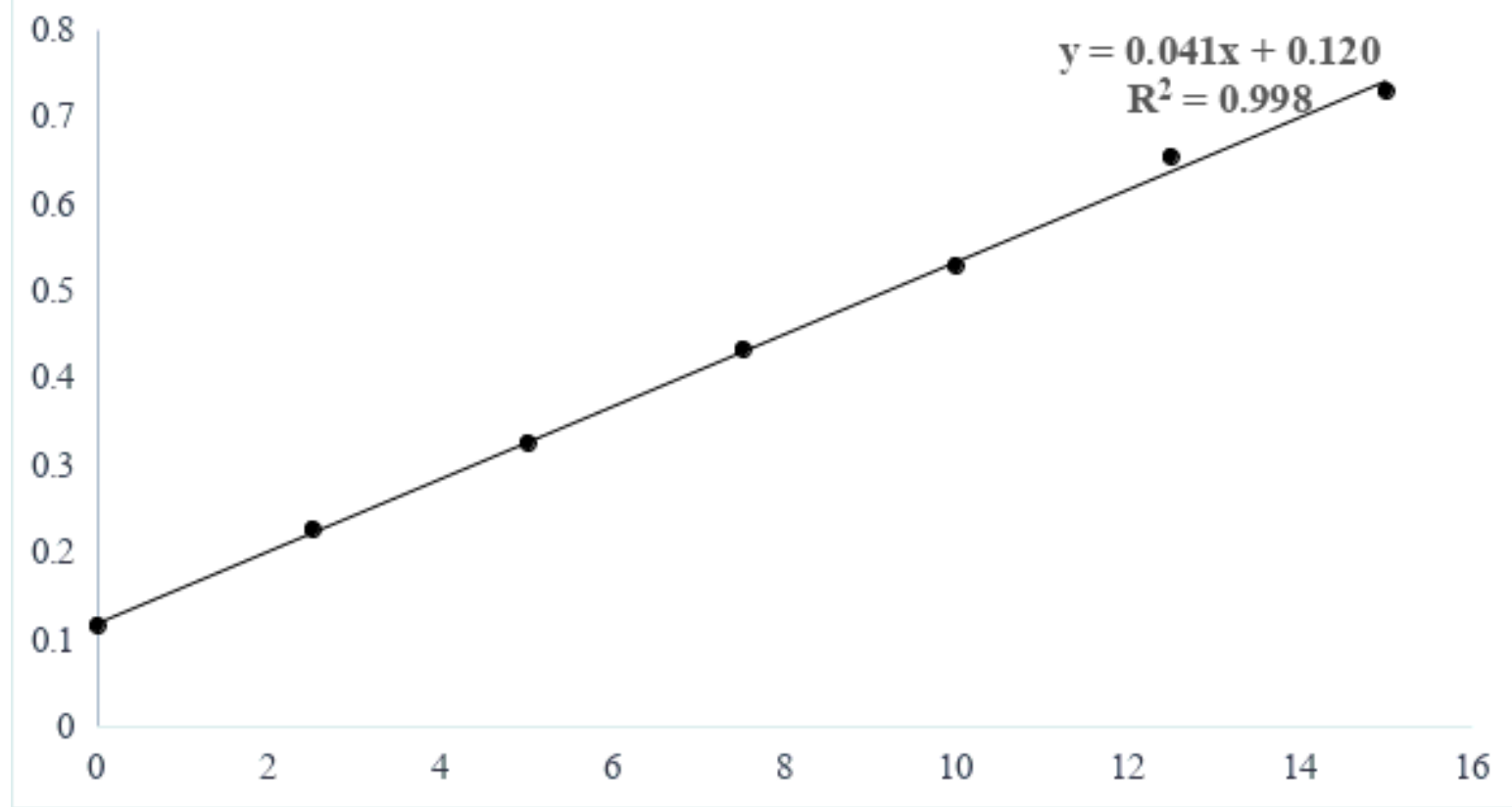

Figure 3

Glucose Standard Curve 


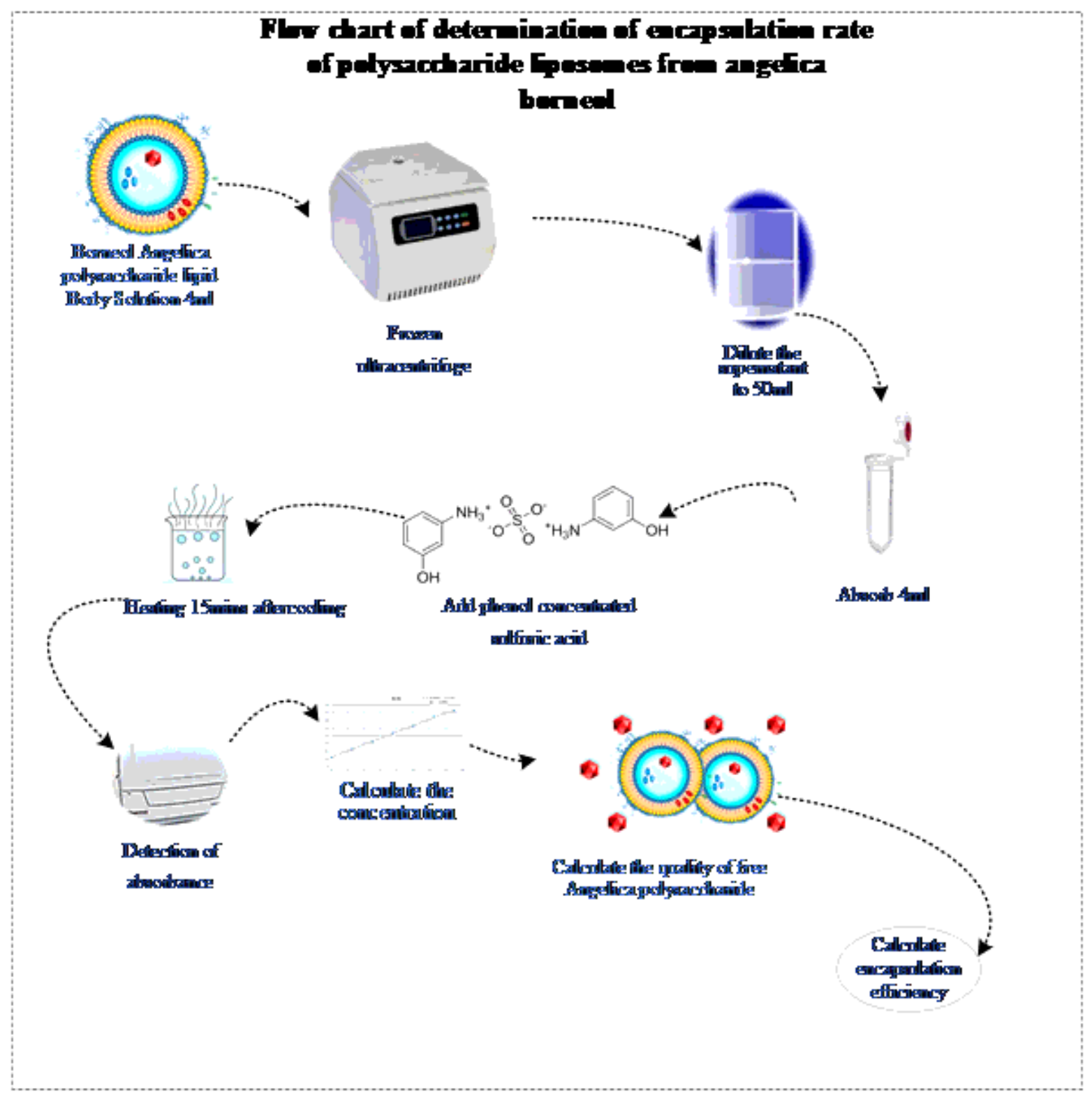

Figure 4

Flow Chart of the BAPL Encapsulation Efficiency Determination

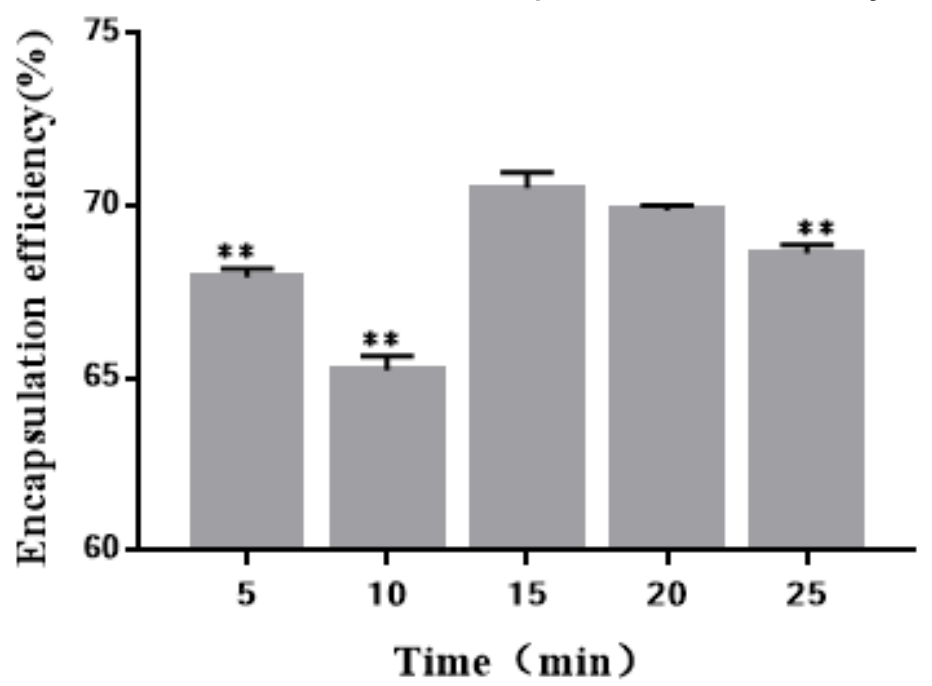




\section{Figure 5}

Effect of Ultrasonic Time on Liposome Encapsulation Efficiency $(X \pm s, n=3)$

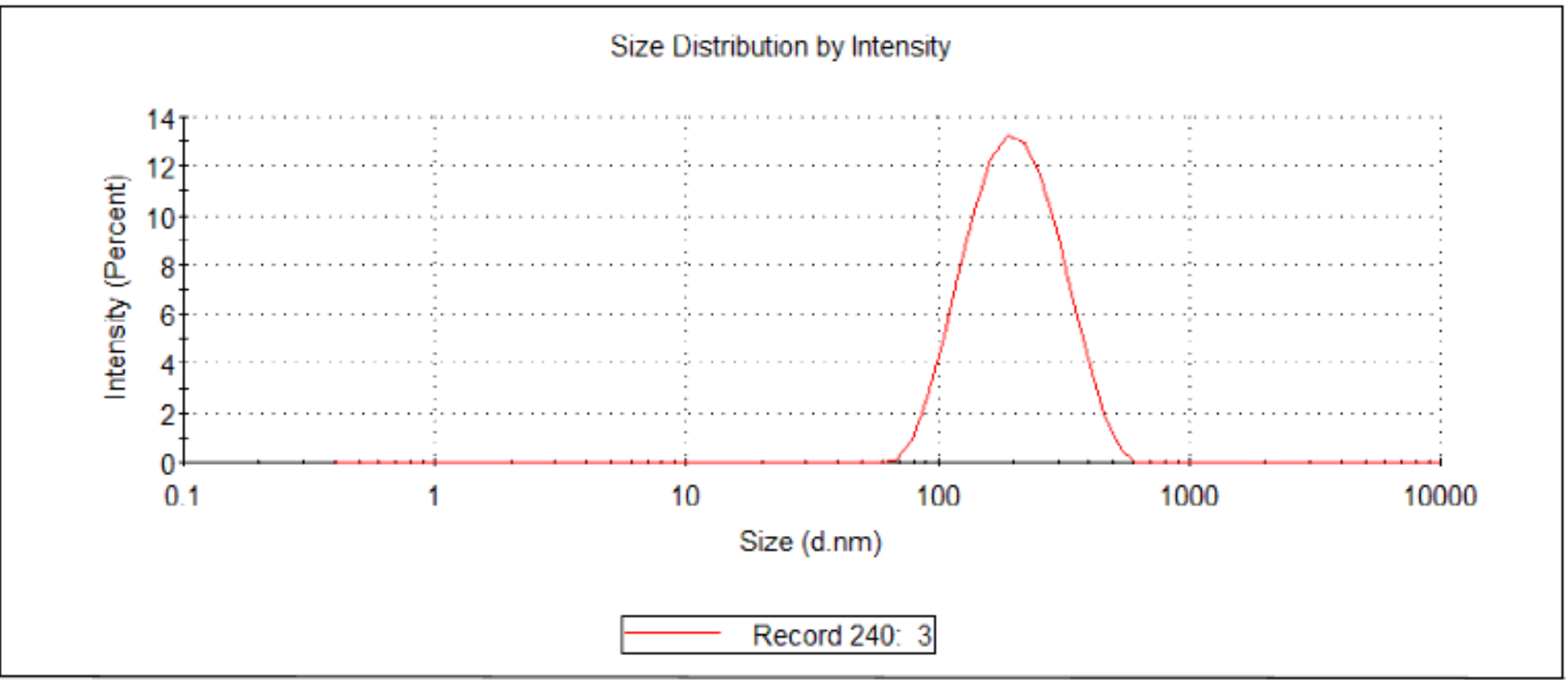

\section{Figure 6}

The Particle Size Distribution of BAPL

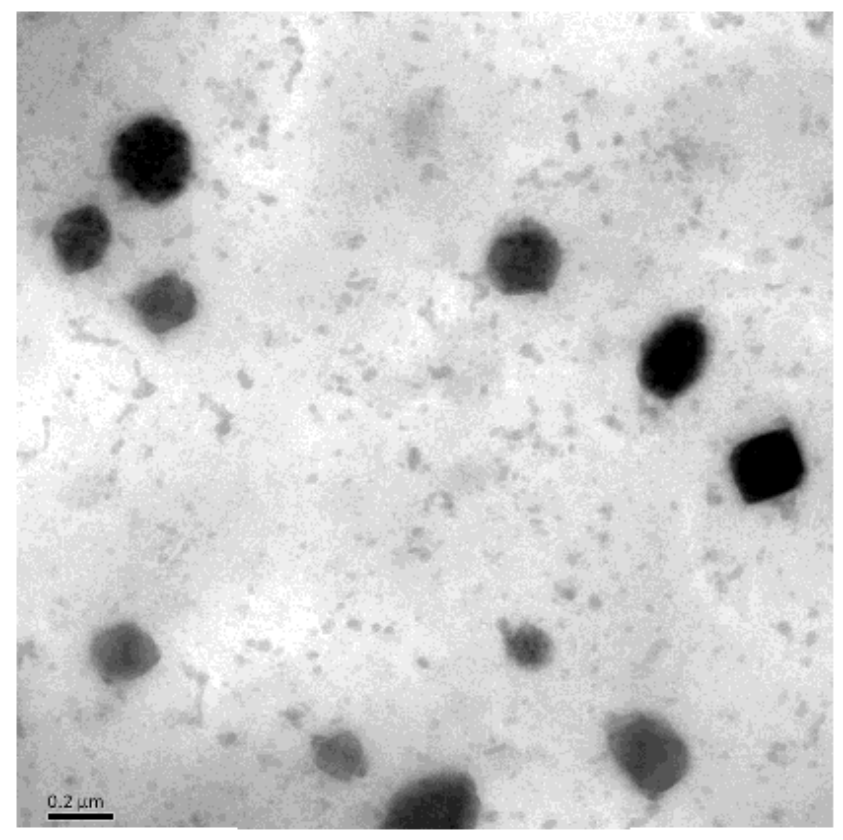

Scale $200 \mathrm{~nm}$

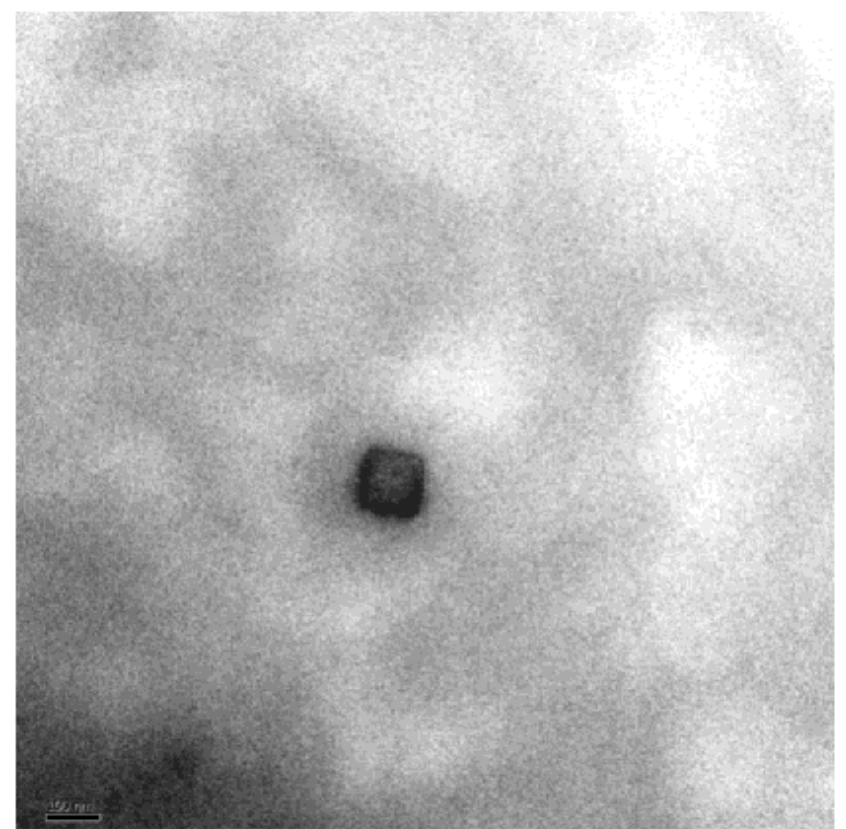

scale $100 \mathrm{~nm}$

Figure 7

Electron Microscopy Analysis of BAPL 


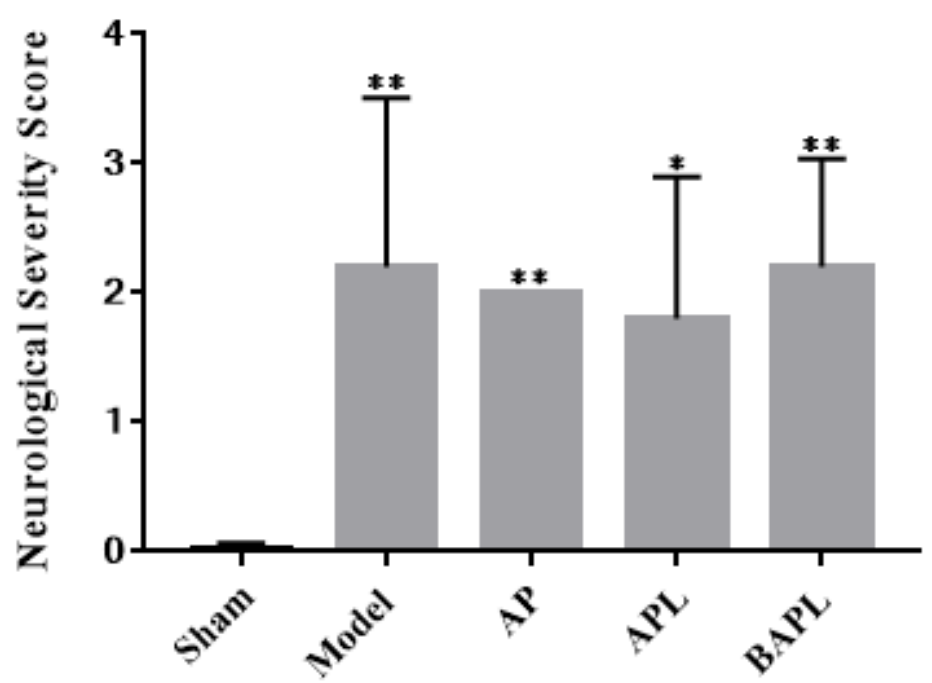

Figure 8

Zea-Longa's Scores of Rats in Each Group $(X \pm s, n=5)$
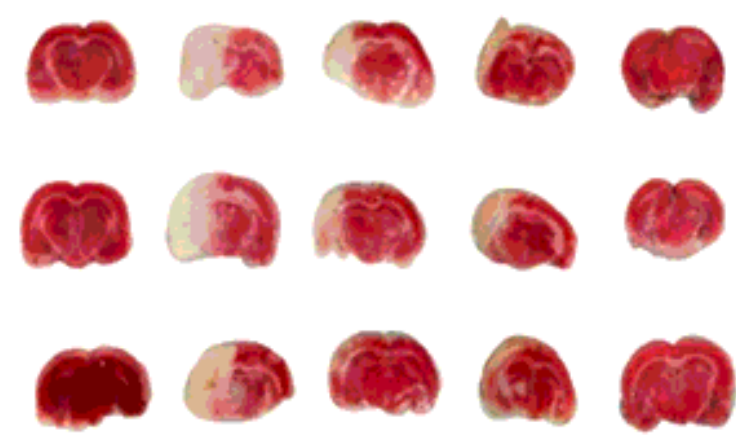

Sham Model AP
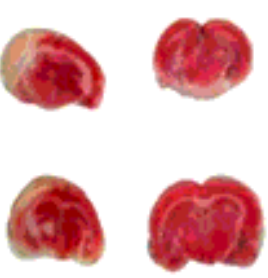

APL

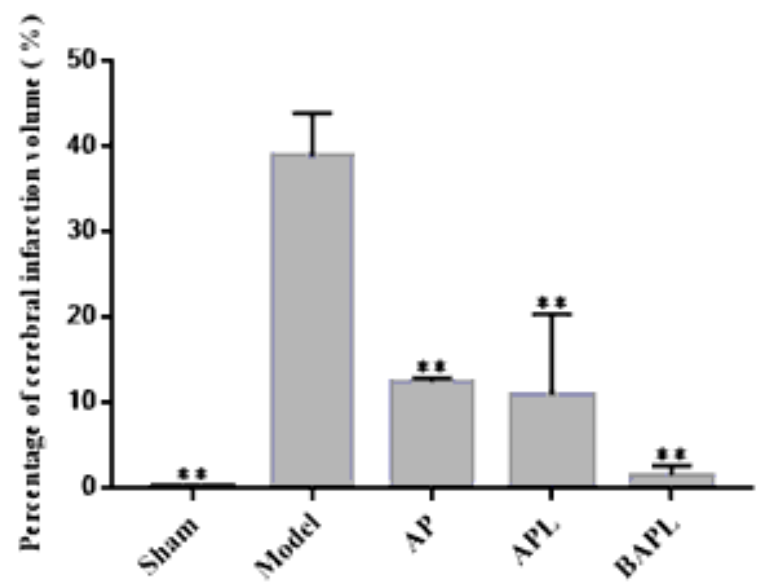

Figure 9

TTC staining results of each group and its cerebral infarction percentage $(X \pm s, n=3)$ 


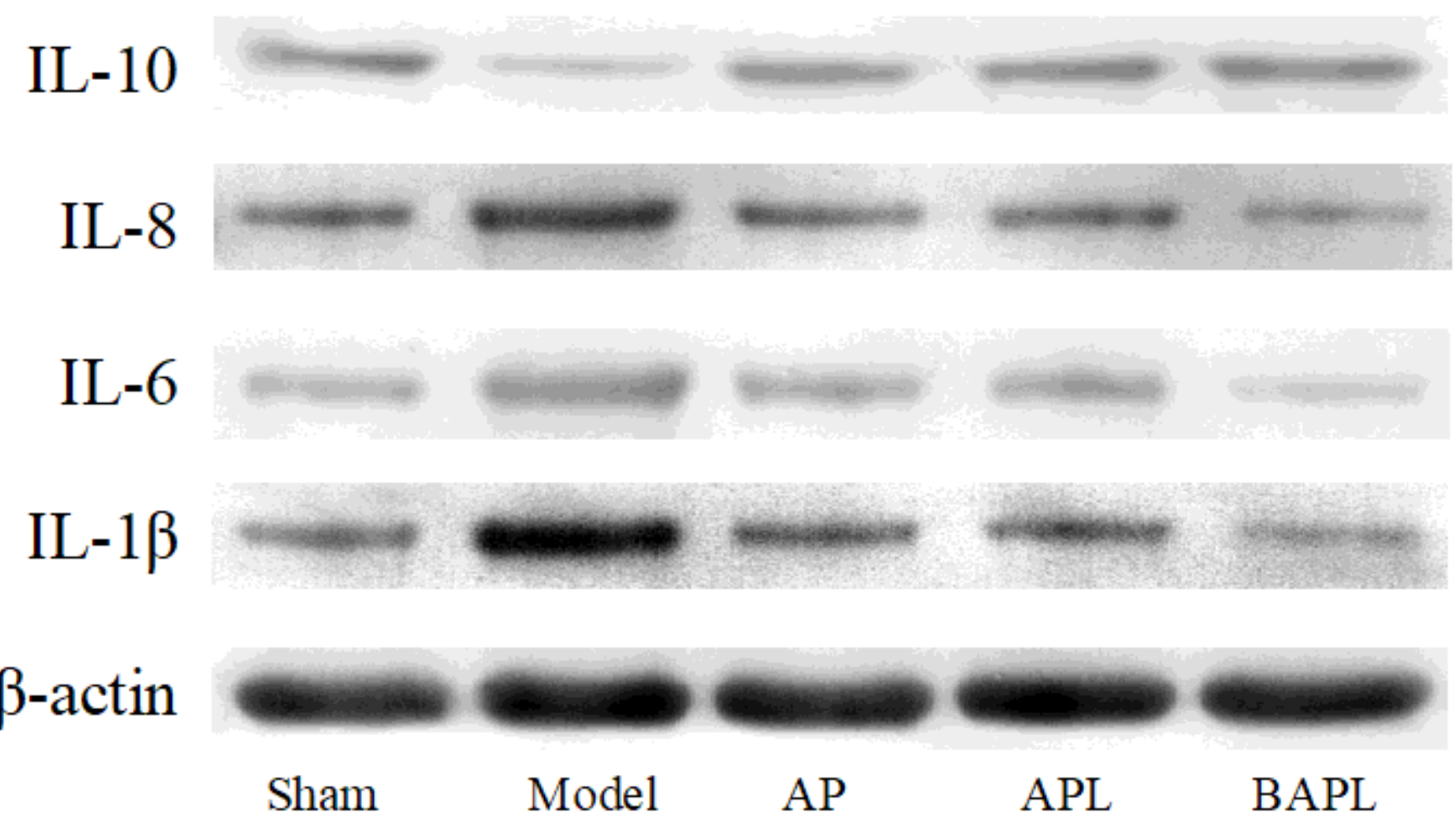

Figure 10

WB analysis of IL-10, IL-8, IL-6 and IL-1 $\beta$ expression levels in rat brain tissue in each group
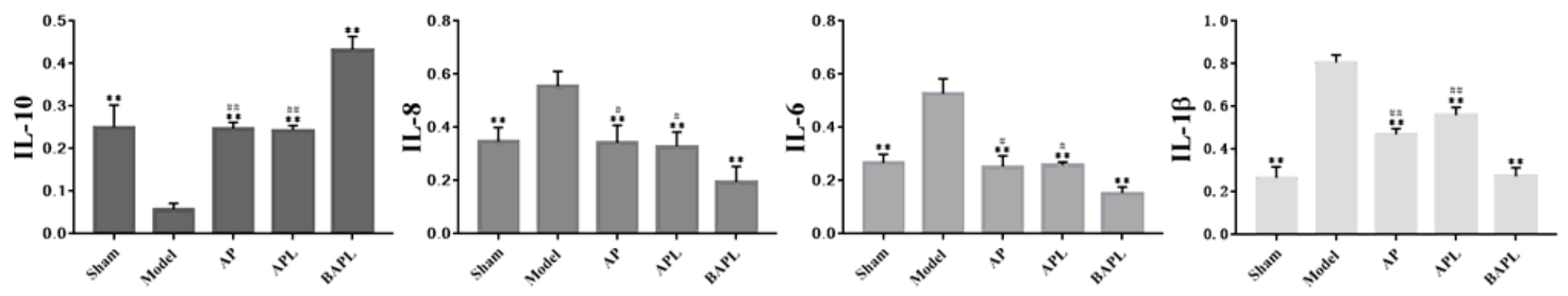

Figure 11

Comparison of the relative values of IL-10, IL-8, IL-6 and IL-1 $\beta$ expression in rat brain tissue from each group $(\mathrm{X} \pm \mathrm{s}, \mathrm{n}=3)$ 


\title{
NF-KBp65
}

\author{
TLR-4
}

ZO-1

$\mathrm{ZO}-2$

$\beta$-actin

Sham Model AP APL BAPL

Figure 12

WB analysis of NF-KBp65, TLR-4, ZO-1 and ZO-2 protein expressions in rats brain tissue of each group
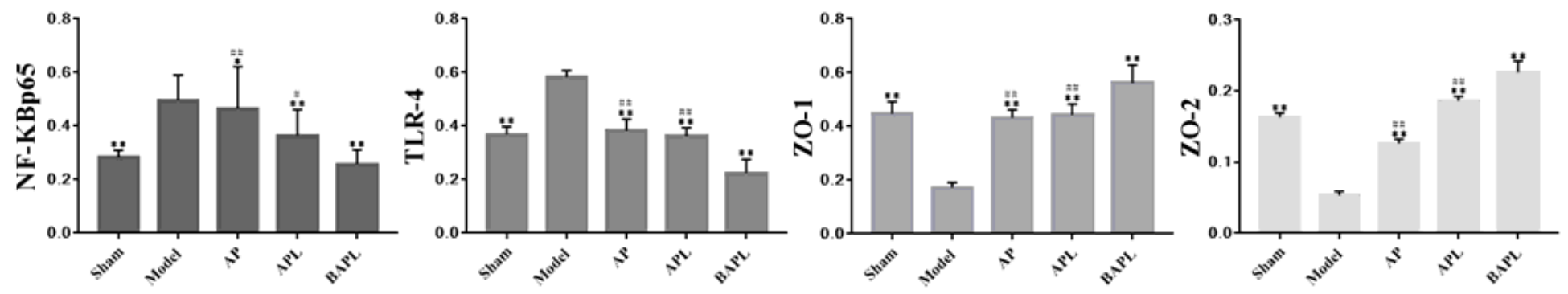

Figure 13

Comparison of the relative values of NF-KBp65, TLR-4, ZO-1, and ZO-2 expression in rat brain tissue from each group $(X \pm s, n=3)$ 


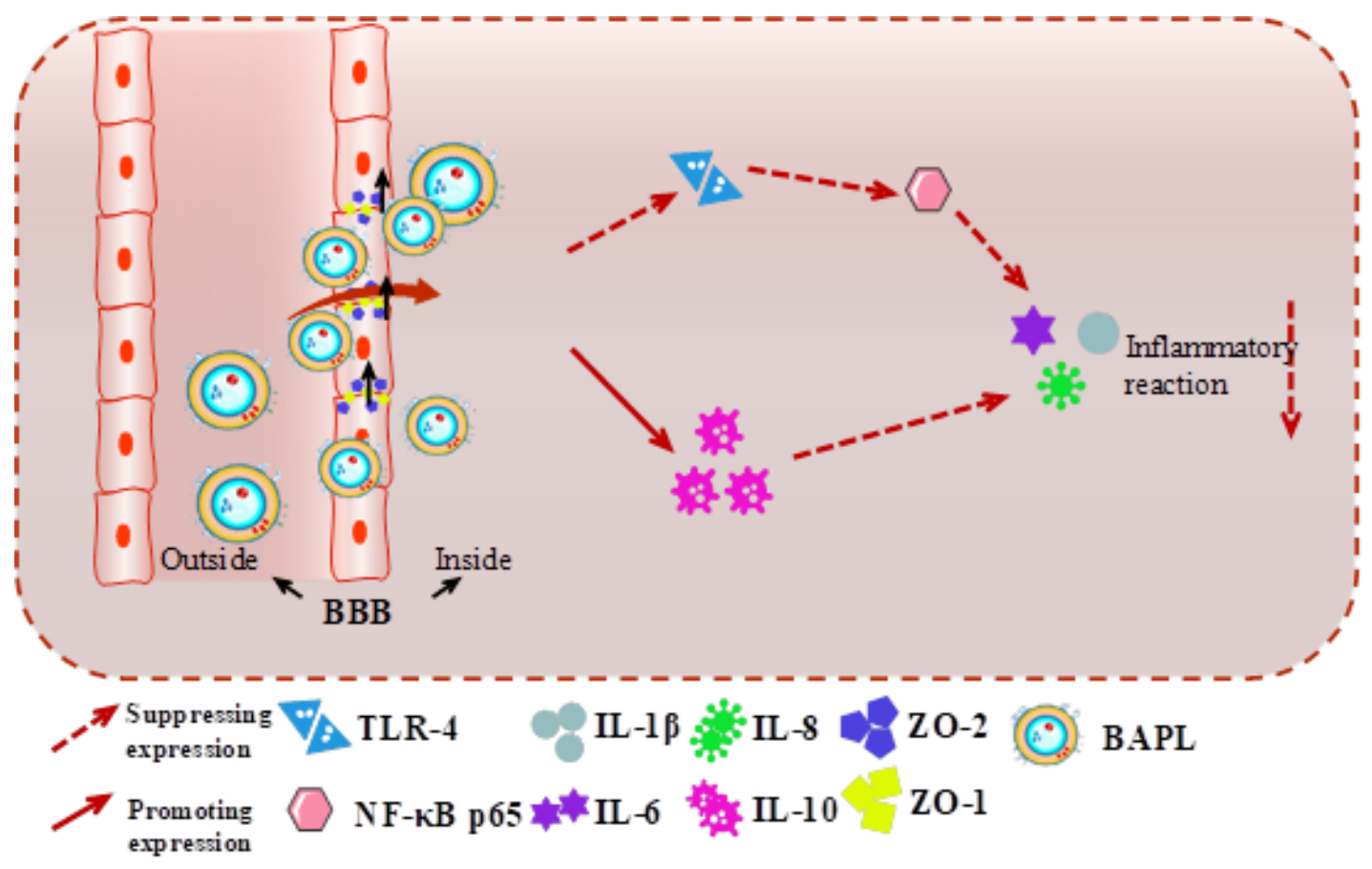

Figure 14

Mechanism of BAPL Anti-Cerebral Ischemia-Reperfusion Inflammatory Reaction 